\title{
PROTOCOLO DE TRANSPLANTE HEPÁTICO PARA METÁSTASE COLORRETAL IRRESSECÁVEL
}

\author{
PROTOCOL FOR LIVER TRANSPLANTATION IN UNRESECTABLE COLORECTAL METASTASIS \\ Lucas ERNANI ${ }^{\oplus}$, Eduardo de Souza Martins FERNANDES ${ }^{2,3}{ }^{\circ}$, Rodrigo Bronze de MARTINO ${ }^{1 \odot}$, \\ Fabricio Ferreira COELHO $^{1}{ }^{\circ}$, Felipe Pedreira Tavares de MELLO $^{2,3}{ }^{\circ}$, Ronaldo ANDRADE ${ }^{2,3}{ }^{\circ}$, \\ Leandro Savattone PIMENTEL ${ }^{2,30}$, Luciana Bertocco de Paiva HADDAD ${ }^{1 \odot}$, Paulo HERMAN ${ }^{1 \odot}$, \\ Wellington ANDRAUS ${ }^{1}$, Luiz Augusto Carneiro D'ALBUQUERQUE ${ }^{1 \oplus}$
}

RESUMO - RACIONAL: O câncer colorretal é a terceira neoplasia mais frequente e metade dos pacientes desenvolvem metástase hepática. O melhor fator prognóstico na metástase hepática de câncer colorretal (MHCCR) é a possibilidade de ressecção com margens livres, porém a maioria permanece irressecável. O racional em realizar transplante hepático $(\mathrm{TH})$ em pacientes portadores de MHCCR está na ampliação do número de pacientes ressecáveis através de uma hepatectomia total. OBJETIVO: Apresentar protocolo brasileiro para realização de transplante hepático em pacientes com MHCCR irressecável. MÉTODO: O protocolo foi realizado por duas instituições com grande volume de ressecções e transplantes hepáticos no Brasil, baseado no trabalho realizado pela Universidade de Oslo. A elaboração foi dividida em 4 etapas. RESULTADO: É apresentada proposta de protocolo para esta doença a ser validada na aplicação clínica. CONCLUSÃO: Foi possível elaborar protocolo de transplante hepático para MHCCR irressecável a fim de uniformizar o tratamento e melhor avaliar os resultados cirúrgicos.

DESCRITORES: Transplante. Transplante de Fígado. Neoplasias Colorretais. Metástase.

ABSTRACT - BACKGROUND: Colorectal cancer (CRC) is the third most common neoplasm, and half of the patients with CRC develop liver metastasis. The best prognostic factor for colorectal liver metastasis (CRLM) is the possibility of performing a resection with free margins; however, most of them remain unresectable. The justification for performing liver transplantation (LT) in patients with CRLM regards an increase in the number of resectable patients by performing total hepatectomy. AIM: The aim of this study was to provide a Brazilian protocol for LT in patients with unresectable CRLM. METHOD: The protocol was carried out by two Brazilian institutions, which perform a large volume of resections and LTs, based on the study carried out at the University of Oslo. The elaboration of the protocol was conducted in four stages. RESULT: A protocol proposal for this disease is presented, which needs to be validated for clinical use. CONCLUSION: The development of an LT protocol for unresectable CRLM aims to standardize the treatment and to enable a better evaluation of surgical results.

HEADINGS: Transplantation. Liver Transplantation. Colorectal Neoplasms. Neoplasm Metastasis.

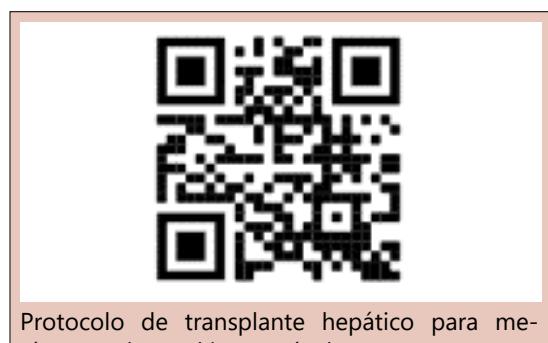

tástase colorretal irressecável

\begin{tabular}{l}
\hline Mensagem central \\
O transplante hepático para pacientes com \\
metástase colorretal irressecável obtém bons \\
resultados quando é realizada seleção pré- \\
operatória restritiva e criteriosa. Este protocolo \\
visa uniformizar esse transplante quando \\
realizado em pacientes com metástase colorretal \\
irressecável.
\end{tabular}

\section{Perspectivas}

Este protocolo visa uniformizar os transplantes hepáticos realizados em pacientes com metástase colorretal irressecável. Dessa forma, uma melhor avaliação dos resultados cirúrgicos, da sobrevida livre de doença e da sobrevida global desses pacientes poderá ser realizada futuramente. Este protocolo está em processo de regulamentação junto ao Sistema Nacional de Transplantes (SNT) do Ministério da Saúde.

Trabalho realizado no 'Departamento de Gastroenterologia, Hospital de Clínicas, Faculdade de Medicina, Universidade de São Paulo, São Paulo, SP, Brasil; ${ }^{2}$ Departamento de Cirurgia Geral e Transplantes, Hospital Adventista Silvestre, Rio de Janeiro, RJ, Brasil; ${ }^{3}$ Serviço de Cirurgia e Transplante de Órgãos Abdominais, Hospital São Lucas - Copacabana, Rio de Janeiro, RJ, Brasil.

Como citar esse artigo: Ernani L, Fernandes ESM, Martino RB, Coelho FF, Mello FPT, Andrade R, Pimentel LS, Haddad LBP, Herman P, Andraus W, D'Albuquerque LAC. Protocolo de transplante hepático para metástase colorretal irressecável. ABCD Arq Bras Cir Dig. 2021;34(4):e1625. https://doi.org//10.1590/0102$672020210002 \mathrm{e} 1625$

Correspondência:

Lucas Ernani.

Email: Icsernani@gmail.com
Financiamento: Não

Conflito de interesses: Não

Recebido 20/12/2020

Aceito $25 / 04 / 2021$ 


\section{INTRODUÇÃO}

$\mathrm{O}$ câncer colorretal (CCR) é a terceira neoplasia mais frequente em ambos os gêneros. No momento do seu diagnóstico, cerca de $25 \%$ dos pacientes apresentam metástase e o fígado é o órgão mais acometido (80\% dos casos). Estima-se que metade dos doentes desenvolverão metástase hepática no curso de sua doença ${ }^{13,14}$.

Atualmente, o tratamento do CCR metastático (estádio IV) se baseia em uma abordagem multidisciplinar e multimodal ${ }^{13,18}$. O melhor fator prognóstico nos portadores de metástase hepática (MHCCR) é a possibilidade de ressecção cirúrgica com margens livres ${ }^{14}$. Neste contexto, a hepatectomia se tornou o principal pilar no tratamento da MHCCR, possibilitando sobrevida global entre 30-55\% em cinco anos e 20-25\% em 10 anos $17,14,18$. Várias estratégias têm sido utilizadas para ampliar a possibilidade de ressecção com remanescente hepático adequado, tais como técnicas de preservação do parênquima, embolização da veia porta, ressecção hepática em dois tempose o ALLPS ("Associating Liver Partition and Portal Vein Ligation for Staged Hepatectomy"). No entanto, mesmo utilizando estas estratégias, a maioria dos pacientes portadores de MHCCR permanecem irressecáveis do ponto de vista funcional ou anatômico 20 .

O racional em realizar transplante hepático $(\mathrm{TH})$ em pacientes portadores de MHCCR está na ampliação do número de pacientes ressecáveis através de hepatectomia total. No entanto, o TH para MHCCR foi considerado contraindicação absoluta pelos resultados inaceitáveis obtidos antes de 1995. A experiência inicial foi relatada pelo European Liver Transplant Registry (ELTR), com taxas de sobrevida de 62\% em 1 ano e $18 \%$ em 5 anos $^{5}$. Vale ressaltar que, no final da década de 80 e início da década de 90, tanto os resultados perioperatórios do TH quanto os quimioterápicos disponíveis para tratamento do CCR justificam esses resultados negativos. Os resultados ruins em panorama de escassez de órgãos culminaram no abandono do TH para MHCCR ${ }^{8,12}$. Baseado nos dados existentes, a atual recomendação da ILTS (International Liver Transplant Society) é realizar o transplante para MHCCR desde que exista protocolo específico ${ }^{11}$.

O objetivo deste estudo foi apresentar proposta de protocolo para guiar o uso clínico do TH na MHCCR. Essa proposta necessita ser validada em estudos posteriores.

\section{MÉTODOS}

O protocolo foi realizado por dois centros com grande volume de ressecções e transplantes hepáticos no Brasil - Hospital de Clínicas da Faculdade de Medicina da Universidade de São Paulo (HC-FMUSP) e Hospital Adventista Silvestre/Hospital São Lucas. A elaboração foi dividida em quatro etapas.

Na primeira etapa foi realizado levantamento dos principais estudos sobre TH em MHCCR até o presente momento. Na segunda, um esboço do protocolo foi realizado pelos dois primeiros autores e pelo último autor baseado no trabalho realizado na Universidade de Oslo ${ }^{4,9}$. Na terceira, 10 especialistas elaboraram a versão final adaptada à realidade brasileira. $\mathrm{Na}$ última etapa do processo foi enviado o trabalho final para aprovação do Sistema Nacional de Transplantes (SNT), vinculado ao Ministério da Saúde.

Serão selecionados centros brasileiros para inclusão no projeto de pesquisa multicêntrico. Um total de 30 pacientes serão incluídos no protocolo de pesquisa, encaminhados via SNT. Os dados pré-operatórios, intra-operatórios e pós-operatórios serão armazenados prospectivamente na plataforma REDCap ${ }^{10}$. Os dados demográficos e pré-operatórios incluírão idade, sexo, IMC, performance clínica, comorbidades, exames laboratoriais, exames de estadiamento, tamanho e número de tumores, realização de quimioterapia prévia, resposta à quimioterapia, anatomopatológico do tumor primário, tempo entre diagnóstico do $\mathrm{CRC}$ e o TH e tipo de TH (cadavérico ou intervivo). Serão analisados também o número de pacientes encaminhados para avaliação da indicação de $\mathrm{TH}$, número de pacientes efetivamente listados (critérios preenchidos) e pacientes excluídos prétransplante (perda de critério). Nos pacientes transplantados será analisado: sobrevida livre de doença e sobrevida global após 1, 3 e 5 anos; protocolo de imunossupressão; episódios de rejeição e necessidade de retransplante.

\section{RESULTADOS}

A Figura 1 mostra o manuseio proposto pelos autores através da criação de um protocolo do TH para MHCCR (Figura 1). A Figura 2 mostra o documento do SNT a ser preenchido para solicitação de situação especial para metástase colorretal irressecável (Figura 2).

\section{DISCUSSÃO}

Nas últimas duas décadas houve progresso na sobrevida após TH em 20-30\%, melhorias nos exames de imagem e introdução de imunossupressores com ação antineoplásica (inibidores do mTOR) ${ }^{15,17}$. Esta evolução técnica aliada ao peculiar panorama de transplantes na Noruega, onde há mais doadores de órgãos do que receptores em lista, proporcionou o cenário ideal para retomar a utilização do TH nas MHCCR. No estudo SECA I, entre 2006 e 2011 na Universidade de Oslo, 21 pacientes foram submetidos a TH para MHCCR; os principais critérios de inclusão na época foram: boa performance clínica (ECOG 0 ou 1), ressecção completa do tumor primário e o mínimo de seis semanas de quimioterapia. Os autores obtiveram sobrevida global de $60 \%$ em cinco anos e identificaram quatro variáveis clínicas associadas a pior prognóstico (critérios de Oslo): diâmetro do tumor $>5.5 \mathrm{~cm}, \mathrm{CEA}>80 \mathrm{ng} / \mathrm{ml}$, intervalo entre ressecção e $\mathrm{TH}<2$ anos e progressão da doença em vigência de quimioterapia 9 .

O mesmo grupo de Oslo deu continuidade à investigação do TH na MHCCR com o estudo SECA II. Entre 2012 e 2016, 15 pacientes foram transplantados com critérios restritivos a fim de se obter resultado semelhante às outras causas de $\mathrm{TH}$. Uma série de critérios foram incluídos para a realização do TH como os critérios de Oslo, irressecabilidade por hepatectomia parcial, resposta radiológica dos tumores após quimioterapia. Os autores obtiveram sobrevida global de $100 \%$ em um ano, $83 \%$ em três anos e $83 \%$ em cinco anos. A sobrevida livre de doença obtida em um ano foi de $53 \%$, em dois anos de $44 \%$ e em três anos de $35 \%$. O principal sítio de recidiva foi pulmonar, sendo a maioria passível de ressecção; portanto, apesar das altas taxas de recorrência, pouca influência teve na sobrevida dos pacientes ${ }^{4}$.

Esses dois estudos noruegueses são marcantes eimportantes pilares no chamado "Transplant Oncology", termo utilizado para denominar o TH como método de tratamento para neoplasias hepatobiliopancreáticas. Recentemente, múltiplos centros na Europa e EUA iniciaram TH para MHCCR ${ }^{11,19}$. Fernandes et al. realizaram, de forma pioneira, o primeiro $\mathrm{TH}$ com doador vivo na América Latina em paciente portador de MHCCR, dentro dos critérios de Oslo ${ }^{6}$

A exclusão do transplante nos casos de tumor de cólon direito e/ou presença de BRAF positivo é um tema a ser discutido. BRAF positivo (mutado) é considerado um fator de risco e 


\begin{tabular}{|c|c|}
\hline \multicolumn{2}{|r|}{ PROTOCOLO DE TRANSPLANTE HEPÁTICO PARA METÁSTASE COLORRETAL IRRESSECÁVEL } \\
\hline ESTRATÉGIA PAF & ENDIMENTO \\
\hline $\begin{array}{l}\text { Local de } \\
\text { atendimento }\end{array}$ & Centros especializados e selecionados pelo SNT \\
\hline $\begin{array}{l}\text { Padronização do } \\
\text { atendimento }\end{array}$ & Realizados dentro de protocolo sob supervisão do SNT para avaliação de resultados \\
\hline $\begin{array}{l}\text { Aprovação do } \\
\text { atendimento }\end{array}$ & $\begin{array}{l}\text { Aprovado em reunião multidisciplinar na instituição de origem com presença obrigatória de radiologista, oncolo- } \\
\text { gista clínico, cirurgião especializado em cirurgia hepatobiliopancreática e cirurgião de transplante de órgãos }\end{array}$ \\
\hline $\begin{array}{l}\text { Notificação do } \\
\text { procedimento }\end{array}$ & Todos os casos devem ser encaminhados à Câmara Técnica Nacional de transplantes para avaliação e aprovação \\
\hline
\end{tabular}

\begin{tabular}{|c|c|}
\hline \multicolumn{2}{|c|}{ INDICAÇÃO PARA O TH } \\
\hline $\begin{array}{l}\text { Seleção dos } \\
\text { pacientes }\end{array}$ & $\begin{array}{l}\text { MHCCR sincrônicas ou metacrônicas, restritas ao fígado, irressecáveis, com um intervalo de tempo entre o diag- } \\
\text { nóstico do tumor primário e a listagem para transplante hepático superior a } 12 \text { ou } 24 \text { meses. }\end{array}$ \\
\hline Autorização oficial & $\begin{array}{l}\text { Após aprovação pela Câmara Técnica Nacional, os pacientes serão incluídos em lista com pontuação especial } \\
\text { (MELD } 30 \text { pontos) }\end{array}$ \\
\hline $\begin{array}{l}\text { Transplantes } \\
\text { intervivos }\end{array}$ & $\begin{array}{l}\text { Seguir os mesmos critérios de inclusão e exclusão dos transplantes cadavéricos } \\
\text { Somente serão cobertos pelo Ministério da Saúde se estiverem dentro dos critérios estabelecidos }\end{array}$ \\
\hline $\begin{array}{l}\text { Critérios de Oslo } \\
\text { (a serem cumpridos } \\
\text { dentro de } 90 \text { dias } \\
\text { antes do TH) }\end{array}$ & $\begin{array}{l}\text { Nível de CEA }<80 \mathrm{ng} / \mathrm{ml} \\
\text { Maior lesão hepática deve ser }<5.5 \mathrm{~cm} \\
\text { Resposta à quimioterapia } \\
\text { Intervalo superior a } 12 \text { ou } 24 \text { meses do diagnóstico de tumor primário de CRC e data de listagem na lista de } \\
\text { transplantes }\end{array}$ \\
\hline
\end{tabular}

\section{CRITÉRIOS DE INCLUSÃO E EXCLUSÃO}

Adenocarcinoma histologicamente confirmado no cólon ou reto

Procedimento cirúrgico padrão com margens de ressecção adequadas do tumor primário, incluindo margens de ressecção circunferencial (CRM) de pelo menos $\geq 2 \mathrm{~mm}$ para pacientes com câncer retal

Metástases hepáticas exclusivas, sincrônicas ou metacrônicas, irressecáveis, não elegíveis para ressecção curativa do fígado após cumprimento dos outros itens expostos

Tratamento prévio com quimioterápico de $1^{\mathrm{a}}$ linha

Antes do início da quimioterapia de primeira linha, nenhuma lesão deve ser maior que $10 \mathrm{~cm}$ e o número total de lesões deve ser de 20 ou menos. Se houver mais de 20 lesões, a maior lesão deve ser de $5 \mathrm{~cm}$ ou menos \begin{tabular}{lll} 
Critérios de inclusão & Resposta à quimioterapia (pelo menos $10 \%$ de resposta de acordo com os critérios RECIST até quimioterapia de \\
\cline { 2 - 3 } (to linha). Os pacientes devem ser aceitos para transplante se não houver progressão da doença em vigência de
\end{tabular} (todos devem estar presentes): quimioterapia

Ausência de sinais de doença metastática extra-hepática ou recidiva local do tumor primário de acordo com TC ou RNM (tórax, abdome e pelve) dentro de 4 semanas antes da reunião multidisciplinar

Ausência de sinais de doença metastática extra-hepática ou recidiva local do tumor primário de acordo com a PET-CT de corpo inteiro dentro de 3 meses antes da reunião multidisciplinar

Colonoscopia normal (nenhum sinal de recidiva local) nos últimos 12 meses antes do transplante

Idade $\geq 18$ anos de idade

Boa performance clínica (ECOG 0 ou 1)

Exames de sangue satisfatórios $\mathrm{Hb}>10 \mathrm{~g} / \mathrm{dl}$, Bilirrubina $<2 \mathrm{x}$ limite superior da normalidade, AST, ALT $<5 \mathrm{x}$ limite superior da normalidade, creatinina $<1,25 \times$ limite superior da normalidade

Perda de peso $>10 \%$ nos últimos 6 meses

Doença metastática extra-hepática prévia ou recidiva local do tumor primário

Pacientes que não receberam tratamento pré-operatório, perioperatório ou pós-operatório padrão para o CRC primário

Critérios de exclusão (apenas um critério Ressecção paliativa do CRC primário (margens comprometidas e/ou presença de menos do que 12 linfonodos é suficiente): avaliados na peça cirúrgica)

$\mathrm{IMC}>35$

Outras doenças malignas nos últimos 5 anos (exceto neoplasia de pele e cervical, as quais serão analisadas pelo anatomopatológico)

Conhecida hipersensibilidade ao inibidor do mTOR (everolimus e/ou sirolimus)

Mulheres grávidas ou amamentando

Recidiva das metástases hepáticas após transplante por metástase colorretal

\section{PÓS-OPERATÓRIO E SEGUIMENTO}

No pós-operatório, os pacientes deverão realizar acompanhamento com consultas ambulatoriais, TC (tórax, Acompanhamento abdome e pelve) e CEA de três e três meses no primeiro ano

clínico Do segundo ano em diante, realizar os mesmos procedimentos de seis em seis meses

\begin{tabular}{|ll|}
\hline IMUNOSSUPRESSÃO, TRATAMENTO DA REJEIÇÃO E RETRANSPLANTE \\
\hline $\begin{array}{l}\text { Imunossupressão } \\
\text { O regime de imunossupressão será realizado de acordo com o esquema institucional de cada equipe } \\
\begin{array}{l}\text { É obrigatório a utilização de inibidor do mTOR (everolimus ou sirolimus) após o primeiro mês ou quando possível } \\
\text { após esse prazo, dependendo das complicações operatórias } \\
\text { rejeição }\end{array}\end{array}$ \\
$\begin{array}{l}\text { Retransplante } \\
\text { hepático por CCH }\end{array}$ & Realizado de acordo com o esquema institucional de cada equipe \\
$\begin{array}{l}\text { Retransplante } \\
\text { nas demais } \\
\text { circunstâncias }\end{array}$ & Permitido em situações como disfunção primária de enxerto, trombose de artéria hepática, rejeição crônica \\
\hline
\end{tabular}

TH=transplante hepático; $\mathrm{MHCCR}=$ metástase hepática câncer colorretal; CCR=câncer colorretal; SNT=Sistema Nacional de Transplantes; TC=tomografia computadorizada; RNM=ressonância nuclear magnética; PET=tomografia por emissão de pósitrons; ECOG=performance status do Eastern Cooperative Oncology Group

Figura 1 - Protocolo criado pelos autores para uso em transplantes hepáticos no tratamento da metástase hepática colorretal irressecável 


\section{SNT SISTEMA NACIONAL DE TRANSPLANTES}

SITUAÇÃO ESPECIAL

METÁSTASE COLORRETAL IRRESSECÁVEL

Paciente:

RGCT:

(n. ${ }^{\circ}$ do registro na Central de Transplantes)

Equipe:

Hospital:

Critério para inclusão em situação especial: MHCCR sincrônicas ou metacrônicas, restritas ao fígado, irressecáveis, com um intervalo de tempo entre o diagnóstico do tumor primário e a listagem para transplante hepático superior a 12 ou 24 meses.

\begin{tabular}{|c|c|}
\hline $\begin{array}{l}\text { Diagnóstico: } \\
\text { ( ) Adenocarcinoma histologicamente confirmado no cólon ou } \\
\text { reto } \\
\text { AP: }\end{array}$ & $\begin{array}{l}\text { Procedimento cirúrgico padrão com margens de ressecção } \\
\text { adequadas do tumor primário, incluindo margens de ressecção } \\
\text { circunferencial (CRM) de pelo menos } \geq 2 \mathrm{~mm} \text { para pacientes com } \\
\text { câncer retal. }\end{array}$ \\
\hline $\begin{array}{l}\text { Metástases hepáticas: } \\
\text { ( ) Metástases hepáticas irressecáveis } \\
\text { ( ) Metástases sincrônicas } \\
\text { ( ) Metástases metacrônicas }\end{array}$ & $\begin{array}{l}\text { Metástases hepáticas exclusivas, sincrônicas ou metacrônicas, } \\
\text { irressecáveis, não elegíveis para ressecção curativa do fígado. } \\
\text { *Antes do início da quimioterapia de } 1^{\text {a }} \text { linha, nenhuma lesão deve } \\
\text { ser maior que } 10 \mathrm{~cm} \text { e o número total de lesões deve ser de } 20 \text { ou } \\
\text { menos. Se houver mais de } 20 \text { lesões, a maior lesão deve ser de } 5 \\
\text { cm ou menos }\end{array}$ \\
\hline $\begin{array}{l}\text { Tamanho da maior lesão* }(\mathrm{cm}): \\
\text { Número máximo de lesões*: }\end{array}$ & Encaminhar relatório médico e laudo dos exames. \\
\hline
\end{tabular}

\begin{tabular}{|c|c|c|c|}
\hline & $\mathrm{IMC} \leq 35$ \\
\hline \multicolumn{3}{|c|}{$\begin{array}{l}\text { Dados clínicos e exames laboratoriais: } \\
\text { IMC: }\end{array}$} & Boa performance clínica (ECOG 0 ou 1) \\
\hline \multicolumn{3}{|c|}{ ECOG: } & Exames de sangue satisfatórios $\mathrm{Hb}>10 \mathrm{~g} / \mathrm{dl}$, Bilirrubina $<2 \mathrm{x}$ limite \\
\hline $\mathrm{Hb}:$ & & Data: / / & superior da normalidade, AST, ALT $<5$ x limite superior da normali- \\
\hline BT: & BD: & Data: / / & dade, creatinina $<1,25 \times$ limite superior da normalidade. \\
\hline AST: & ALT: & Data: / / & Encaminhar relatório médico e laudo dos exames. \\
\hline Cr: & & Data: / / & \\
\hline CEA: & & Data: / / & \\
\hline
\end{tabular}

\begin{tabular}{|c|c|c|}
\hline $\begin{array}{l}\text { Estadiamento: } \\
\text { TC (T/A/P) } \\
\text { RNM (abdome) } \\
\text { PET-CT } \\
\text { Colonoscopia }\end{array}$ & $\begin{array}{lll}\text { Data: } & / & / \\
\text { Data: } & / & / \\
\text { Data: } & / & / \\
\text { Data: } & / & /\end{array}$ & $\begin{array}{l}\text { Estadiamento da doença realizada por TC (tórax, abdome e pelve), } \\
\text { RNM (abdome), PET-CT e colonoscopia. } \\
\text { Ausência de sinais de doença metastática extra-hepática ou } \\
\text { recidiva local do tumor primário de acordo com TC ou RNM (tórax, } \\
\text { abdome e pelve) dentro de } 4 \text { semanas antes da reunião multidis- } \\
\text { ciplinar. } \\
\text { Ausência de sinais de doença metastática extra-hepática ou reci- } \\
\text { diva local do tumor primário de acordo com a PET-CT de corpo } \\
\text { inteiro dentro de } 3 \text { meses antes da reunião multidisciplinar. } \\
\text { Colonoscopia normal (nenhum sinal de recidiva local) nos últimos } \\
12 \text { meses antes do transplante. } \\
\text { Encaminhar relatório médico e laudo dos exames. }\end{array}$ \\
\hline
\end{tabular}

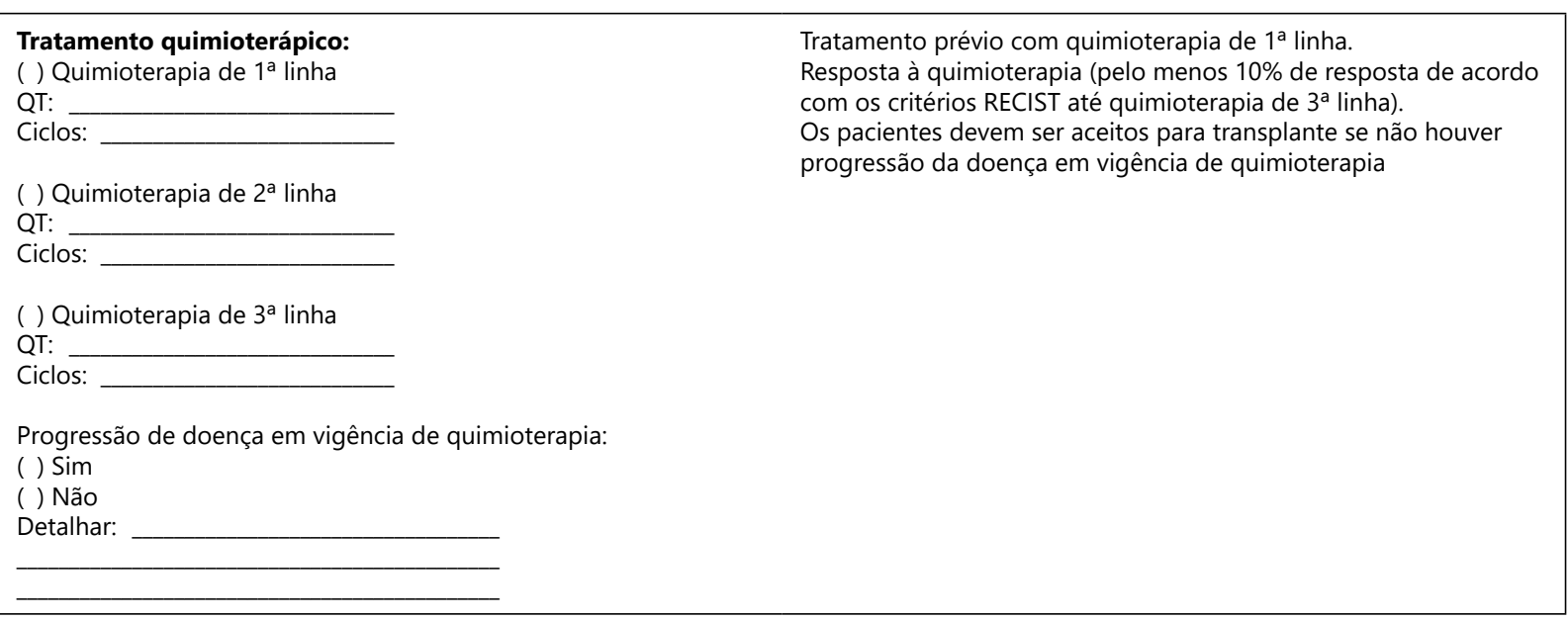

\section{Critérios de Oslo:}

( ) Nível de CEA <80 ng / ml

Obrigatoriamente devem ser cumpridos dentro de 90 dias antes

( ) Maior lesão hepática deve ser $<5.5 \mathrm{~cm}$ do TH

( ) Resposta à quimioterapia

( ) Intervalo $>12$ ou 24 meses do diagnóstico de tumor primário

de CRC e data de listagem na lista de transplantes

Assinatura e Carimbo do Médico:

Data:

TH=transplante hepático; $\mathrm{MHCCR}=$ metástase hepática câncer colorretal; CCR=câncer colorretal; SNT=Sistema Nacional de Transplantes; TC=tomografia computadorizada;

RNM=ressonância nuclear magnética; $\mathrm{PET}=$ tomografia por emissão de pósitrons; $\mathrm{ECOG}=$ performance status do Eastern Cooperative Oncology Group; $\mathrm{QT}=$ quimioterapia

Figura 2 - Documento do Sistema Nacional de Transplantes (SNT) a ser preenchido para solicitação de situação especial para metástase colorretal irressecável 
com pior resultado após o transplante. Os tumores de cólon direito também apresentam pior prognóstico, justamente por apresentarem maior frequência de BRAF positivo ${ }^{16}$. Os atuais estudos clínicos em andamento são heterogêneos quanto a estes itens e, portanto, optamos por manter em nosso protocolo até maiores evidências. Na Noruega (NCT01479608, NCT02215889, NCT03494946) e na Alemanha (NCT03488953), os estudos não adotam estes critérios para exclusão; na França (NCT02597348), Canadá (NCT02864485) e Itália (NCT03803436), BRAF positivo é um critério de exclusão ${ }^{3,11}$.

O protocolo ora apresentado está em processo de regulamentação pelo SNT para vigência em todo o território nacional ${ }^{2}$.

\section{CONCLUSÃO}

Foi elaborado protocolo de transplante hepático para metástase colorretal irressecável a fim de uniformizar o tratamento e melhor avaliar os resultados cirúrgicos, de sobrevida livre de doença e da sobrevida global desses pacientes.

\section{AGRADECIMENTOS}

Os autores agradecem de modo especial a Eduardo Fernandes e Pål-Dag Line, os quais foram a base para realização deste trabalho.

\section{REFERÊNCIAS}

1. Adams RB, Aloia TA, Loyer E, Pawlik TM, Taouli B, Vauthey JN; Americas Hepato-Pancreato-Biliary Association; Society of Surgical Oncology; Society for Surgery of the Alimentary Tract. Selection for hepatic resection of colorectal liver metastases: expert consensus statement. HPB (Oxford). 2013;15(2):91-103. doi: 10.1111/j.14772574.2012.00557.x.

2. Brasil. Decreto n. 2.268, de 30 de junho de 1997. Regulamenta a Lei $\mathrm{n}$. 9.434, de 4 de fevereiro de 1997, que dispõe sobre a remoção de órgãos, tecidos e partes do corpo humano para fins de transplante e dá outras providências. Brasília: Diário Oficial da União 1 jul 1997; (1):13739.

3. ClinicalTrials.gov [Internet]. Bethesda (MD): U.S. National Library of Medicine; 2002. Available from: https://clinicaltrials.gov/ct2/ home;

4. Dueland S, Syversveen T, Solheim JM, Solberg S, Grut H, Bjørnbeth BA, Hagness M, Line PD. Survival Following Liver Transplantation for Patients With Nonresectable Liver-only Colorectal Metastases. Ann Surg.2020;271(2):212-218. doi: 10.1097/SLA.0000000000003404.

5. European Liver Transplant Registy (ELTR). Data Analysis Booklet. Paris. 2007.

6. Fernandes ESM, Line PD, Mello FP, Andrade RO, Girão CL, Pimentel LS, César C, Suleiman TS, Waechter FL, Oliveira ATT, Torres OJM. Living donor liver transplant for colorectal liver metastasis: the first case in Latin America. Arq Bras Cir Dig. 2019; 32(4):e1468. doi: 10.1590/0102-672020180001e1468.

7. Fong Y, Fortner J, Sun RL, Brennan MF, Blumgart LH. Clinical score for predicting recurrence after hepatic resection for metastatic colorectal cancer: analysis of 1001 consecutive cases. Ann Surg. 1999; 230(3):309-18; discussion 318-21. doi: 10.1097/00000658199909000-00004.

8. Foss A, Adam R, Dueland S. Liver transplantation for colorectal liver metastases: revisiting the concept. Transpl Int. 2010;23(7):679-85. doi: 10.1111/j.1432-2277.2010.01097.x.

9. Hagness M, Foss A, Line PD, Scholz T, Jørgensen PF, Fosby B, Boberg KM, Mathisen O, Gladhaug IP, Egge TS, et al. Liver transplantation for nonresectable liver metastases from colorectal cancer. Ann Surg. 2013; 257(5):800-6. doi: 10.1097/SLA.0b013e3182823957.

10. Harris PA, Taylor R, Thielke R, Payne J, Gonzalez N, Conde JG. Research electronic data capture (REDCap)--a metadata-driven methodology and workflow process for providing translational research informatics support. J Biomed Inform. 2009 Apr;42(2):37781. doi: 10.1016/j.jbi.2008.08.010.

11. Hibi T, Rela M, Eason JD, Line PD, Fung J, Sakamoto S, Selzner N, Man K, Ghobrial RM, Sapisochin G. Liver Transplantation for Colorectal and Neuroendocrine Liver Metastases and Hepatoblastoma. Working Group Report From the ILTS Transplant Oncology Consensus Conference. Transplantation. 2020; 104(6):1131-1135. doi: 10.1097/TP.0000000000003118.

12. Hoti $\mathrm{E}$, Adam R. Liver transplantation for primary and metastatic livercancers. Transpl Int.2008;21(12):1107-17; doi: 10.1111/j.14322277.2008.00735.x

13. Krüger JAP, Fonseca GM, Makdissi FF, Jeismann VB, Coelho FF, Herman P. Evolution in the surgical management of colorectal liver metastases: Propensity score matching analysis (PSM) on the impact of specialized multidisciplinary care across two institutional eras. J Surg Oncol. 2018; 118(1):50-60; doi: 10.1002/jso.25098.

14. Lee RM, Cardona K, Russell MC. Historical perspective: Two decades of progress in treating metastatic colorectal cancer. J Surg Oncol. 2019;119(5):549-563; doi: 10.1002/jso.25431.

15. Lima AS, Pereira BB, Jungmann S, Machado CJ, Correia MITD. Risk factors for post-liver transplant biliary complications in the absence of arterial complications. Arq Bras Cir Dig. 2020;33(3):e1541. doi: 10.1590/0102-672020200003e1541.

16. Line PD, Dueland S. Liver transplantation for secondary liver tumours: The difficult balance between survival and recurrence. J Hepatol.2020;73(6):1557-1562. doi: 10.1016/j.jhep.2020.08.015.

17. Pinto LEV, Coelho GR, Coutinho MMS, Torres OJM, Leal PC, Vieira CB, Garcia JHP. Risk factors associated with hepatic artery thrombosis: analysis of 1050 liver transplants. Arq Bras Cir Dig. 2021;33(4):e1556. doi: 10.1590/0102-672020200004e1556.

18. Raoof M, Haye S, Ituarte PHG, Fong Y. Liver Resection Improves Survival in Colorectal CancerPatients: Causal-effects From Populationlevel Instrumental Variable Analysis. Ann Surg. 2019; 270(4):692700. doi: $10.1097 /$ SLA. 0000000000003485.

19. Sapisochin G, Hibi T, Ghobrial M, Man K. The ILTS Consensus Conferenceon TransplantOncology:Setting theStage. Transplantation. 2020; 104(6):1119-1120. doi: 10.1097/TP.0000000000003175.

20. Schadde E, Grunhagen DJ, Verhoef C, Krzywon L, Metrakos P. Limitations in resectability of colorectal liver metastases 2020 A systematic approach for clinicians and patients. Semin Cancer Biol. 2021;71:10-20. doi: 10.1016/j.semcancer.2020.09.008. 